The oblate and prolate shells were each of them made from the two hemispheres of sheet copper which plumbers solder together to make their globular floaters. By a littie hammering it is easy to alter the hemispheres to the proper shapes to make either the prolate or the oblate figure.

Theory had pointed out that the rotation of a liquid in a rigid shell of oval figure, being a configuration of maximum energy for given vorticity, would be unstable if the containing vessel is left to itself supported on imperfectly elastic supports, although it would be stable if the vessel were held absolutely fixed, or borne by perfectly elastic supports, or left to itself in space unacted on

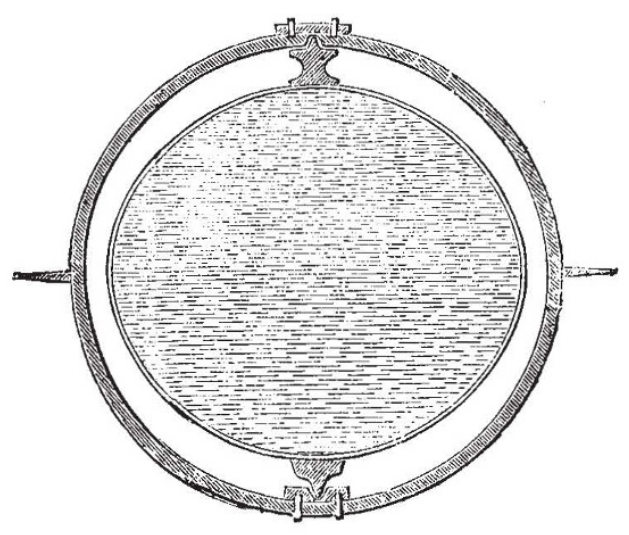

by external force; and it was to illustrate this theory that the cyal shell was made and filled with water and placed in the apparatus. The result of the first trial was literally startling, although it ought not to have been so, as it was merely a realisatien of what had been anticipated by theory. The framework was held as firmly as possible by one person with his two hands, keeping it as steady as he could. The spinning by means of a fine cord ${ }^{1}$ round a small $\mathrm{V}$ pulley of $\frac{1}{3}$-inch diameter on the axis of the oval shell, and passing round a large fly-wheel of 3 feet diameter turned at the rate of about one round per sicond, was continued for several minutes. This in the case of the oblate shell, as was known from previous experiments, would have given amply sufficient rotation to the contained water to cause the apparatus to act with great firmness like a solid gyrostat. In the first experiment with the oval shell the shell was seen to be rotating with great velocity during the last minute of the spinning; but the moment it was released from the cord, and when, ho!ding the framework in my hands, I commenced carrying it towards the horizontal glass table to test its gyrostatic quality, the framework which I he'd in my hands gave a violent niacontrollable lurch, and in a few seconds the shell stopped turning. I saw that one of the pivots had become bent over, by yielding of the copper shell in the neighbourhood of the stiff pivot-carrying disk, soldered to it, showing that the liquid had exerted a very strong couple against its containing shell, in a plane through the axis, the effort to resist which by my hands had bent the pivot. The shell was refitted with more strongly attached pivots, and the experiment has been repeated several times. In every case a decided uneasiness of the framework is perceived by the person holding it in his hands during the suning; and as soon as the cord is cut and the person holding it carries it towards the experimental table, the framework bagins, as it were, to wriggle round in his hands, and by the time the framework is placed on the table the rotation is nearly all gone. Its utter failure as a gyrostat is precisely what was expected from the theory, and presents a truly wonderful contrast from what is obierved with the apparatus and operations in every respect similar, except having an oblate instead of a prolate shell to contain the liquid.

I Instead of using a long cord first w. und on a bobbin, and finally wound up in the circumference of the large wheel as described in NATURE, February $x, 1877$, p. 297 , I have since found it much more convenient to use an endless cord little more than half round the crrcumference of the large wheel, and less than half round the circumference of the $V$ pulley of the gyrustat: and $t$, keep it tight enough to exert whatever tangential torce on the $\mathrm{V}$ pulley is desired by the ferson holding the framework in his hand. After continuing the spinning by turning the fly-wheel for as long a time as is judged proper, the endless cyrd is cut with a pair of sciscors and the gyrostat released.

\section{ON A DISTURBING INFINITY IN LORD RAYLEIGH'S SOLUTION FOR WAVES IN A PLANE VORTEX STRATUM}

IN the paper in last week's NATURE under this heading by Sir William Thomson, the lower part of the illustration was inadvertently turned round at the last moment by the printer ; the cut should stand as follows :-
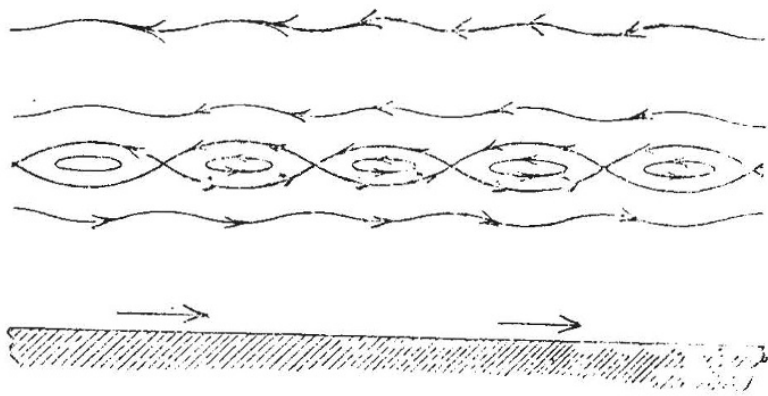

\section{SARGASSL:M 1}

THIS paper opens with a discuscion of the value of the species Sargassum bacciferum, the particular species of this genus which is well known as the Gulf-weed. The author considers that the floating plants to which this namej has been given are simply fragments of many varieties or species of Sargassum, more particularly of $S$. vulgare. In support of this; view he points out that, from the accounts of nearly all authors who have examined specimens, it appears that the lower part of the stem had been broken across, and that it is therefore fair to conclude that they belong to plants which are rooted under ordinary circumstances. This conclusion had been already arrived at by Rumphius, C. Agardh, Rennell, Humboldt, and more recently by G. von Martens; but of the e writers Iiennell and Humboldt are of opinion that the floating fragments continue to grow, and in this they agree with Thunberg, Meyen, and Harvey. Dr. Kuntze contends that there is not sufficient evidence forthcoming to establish the correctness of this view. He urge; that, even admitting that some growth takes place, it is only temporary, and that it therefore affords no ground for regarding these as pelagic plants. The only other cases of growth of Fuci when floating are offered by Macrocystis pyrifera (Sir Joseph (Mr. Moseley, "Notes by a Naturalist ond by Fucus vesiculosus doubtless Dr. "Notes by a Naturalist on the Challenger"), and question naturally arises as to whether these floating plants are actively living, or are dying, or dead.

In the case of Sargassum Dr. Kur tze considers that their bright yellow colour is due to changes taking place, either preliminary to or in consequence of deatb, in the brown colouring-mat:er of the attached forms to which he believes the floating fragments belong. Mr. Moseley, however, is of opinion that this is the natural colour of these plants whilst living. It does not appear that any such difference in colour has been noticed in attached and floating specimens of Macrocystis or of Fucus, and this is a fact which is not in harmony with Dr. Kuntze's views respecting Sargassum. Again, the general ob:ervation that these floating Fuci have no reproductive nrgans offers a further difficulty which they do not explain. Dr. Kuntze endeavours to meet the diffi. culty by stating that he has found receptacles occasionally in free-swimming individuals, and he gives figures of two plants bearing them; but neither from the fizures nor from his account of them is it possible to conclude with certainty that the bodie: in question are really of a repruductive nature; and he explains the usual absence of the e organs in the floating individuals by suggesting that the receptacles, being the most fragile parts of the plant, are the most readily destroyed, and further that, owing to the small number of air-chambers with which they are provided, they would sink on becoming detached. In this case, as in that of the colour, these explanations respecting Sargassum will only become valid when they are found to hold good of Macrocystis and of Fucus also. It is apparent that the

$x$ "Revision von Sargas-um und das sogenannte Sargasso-Meer." Von Dr. Otto Kuntze (Engler's botanische faibrbïcher, Bd. i. Heft iii., I $8 \&$ ). Leipzig, Engelmarn.) 